\title{
Tako-tsubo cardiomyopaty after femur fracture
}

\author{
Davide Lazzarini ${ }^{1^{*}}$, Cesare Cangiotti ${ }^{1}$, Luca Morolli ${ }^{1}$, Sante Morelli ${ }^{1}$, Jonathan Montomoli ${ }^{1}$, \\ Giorgio Ioli $^{1}$, Paola Venturi ${ }^{2}$, Giancarlo Piovaccari ${ }^{2}$ \\ ${ }^{1}$ The Third Department of Internal Medicine, Santarcangelo di Romagna, Azienda USL di Rimini, Rimini, Italy; \\ *Corresponding Author: davide.lazzarini@auslrn.net \\ ${ }^{2}$ Cardiovascular Diseases Department, Ospedale “Infermi” Rimini, Azienda USL di Rimini, Rimini, Italy
}

Received 16 November 2011; revised 7 December 2011; accepted 14 January 2012

\begin{abstract}
We present the case of an 86-year-old woman referred to us from Orthopaedics Division after surgical treatment for femur fracture without personal or family history of ischemic cardiopathy. During hospitalization the patient had stomach-ache and the electrocardiogram demonstrated ST-segment elevation in anterior leads. The coronarography showed haziness, but no critical stenosis; ventricolography revealed apical ballooning of the left ventricle with severe systolic dysfunction. She was treated with dopamine in renal dose, beta-blockers and warfarin. When she was in fairly good condition, she started physiotherapy for endoprosthesis for the femur fracture. Clinicians should consider takotsubo cardiomyopathy in the differential diagnosis of patients presenting with chest pain, especially in women with a recent history of physical stress.
\end{abstract}

Keywords: Takotsubo Cardiomyopathy; Acute Coronary Syndrome; Apical Ballooning Syndrome; Left Ventricular Disfunction; Broken-Heart Syndrome

\section{INTRODUCTION}

Non ischaemic transient apical balloning is an heart disorder which mimics an acute coronary event, of unknown origin, described the first time by Sato et al. [1] and named takotsubo because of its resemblance to a Japanese fishing pot in ventriculography. The syndrome consists of chest discomfort with acute onset, transient and usually reversible wall motion abnormalities of the left ventricular apex, electrocardiographic changes resembling acute myocardial ischemia and release of myocardial enzymatic biomarkers mimicking acute coronary syndrome in patients without significant coronary artery stenosis. Patients are predominantly postmenopausal fe- males with a recent, emotionally stressful event.

\section{CASE REPORT}

A 86-year-old woman was admitted to Emergency Department for a intertrochanteric fracture of the right femur due to a fall for a lipothymia. The patient had been diagnosed for major depressive disorder, Parkinson's syndrome, osteoporosis and she had a previous surgical treatment for vulvar epidermoid cancer. There was no personal or family history of ischemic cardiopathy; in Picture 1 there's an electrocardiogram performed in 2007. A cranial computed tomography scan showed cerebral atrophy. The chest X-ray and the electrocardiogram were normal. Two days after hospitalization in the Orthopaedics Division she was operated of endoprosthesis for the femur fracture. During the postoperative clinical course the haemoglobin was $7.4 \mathrm{~g} / \mathrm{dl}(\mathrm{NV}=12 \mathrm{~g} / \mathrm{dl})$ and she received $2 \mathrm{U}$ of concentrated red blood cells; in the fourteenth day she was transferred to our post-acuity ward for physiotherapy.

In our ward haemoglobin was $11.7 \mathrm{~g} / \mathrm{dl}$, blood pressure 105/55 mmHg, heart rate $90 \mathrm{bpm}$ and she started physiotherapy. In the third day she had stomach ache regressed by intravenous administration of ranitidine hydrochloride $100 \mathrm{mg}$. One hour later the patient was diaphoretic, the blood pressure was 60/40 $\mathrm{mmHg}$, she referred thoracic pain and the electrocardiogram showed in Picture 2 had heart rate of $90 \mathrm{bpm}$, ST sopraelevation in DI, $\mathrm{V}_{2}, \mathrm{~V}_{3}$ and $\mathrm{V}_{4}$. T-troponin test was $453 \mathrm{ng} / \mathrm{L}(\mathrm{NV}<$ $10 \mathrm{ng} / \mathrm{L}$ ); the intra arterial gas analysis is showed in Table 1.

A second T-Troponin test performed 4 hours later revealed $1227 \mathrm{ng} / \mathrm{L}(\mathrm{NV}<10 \mathrm{ng} / \mathrm{L})$. An intravenous administration of $300 \mathrm{mg}$ of lysine acetylsalycilate was done and the patient was urgently transferred in cardiovascular ward for an acute coronary syndrome.

In the Cardiovascular Disease Department an echocardiography was performed, which showed left ventricular systolic disfunction with apical akinesis and ejection fraction decreased to $35 \%$. 


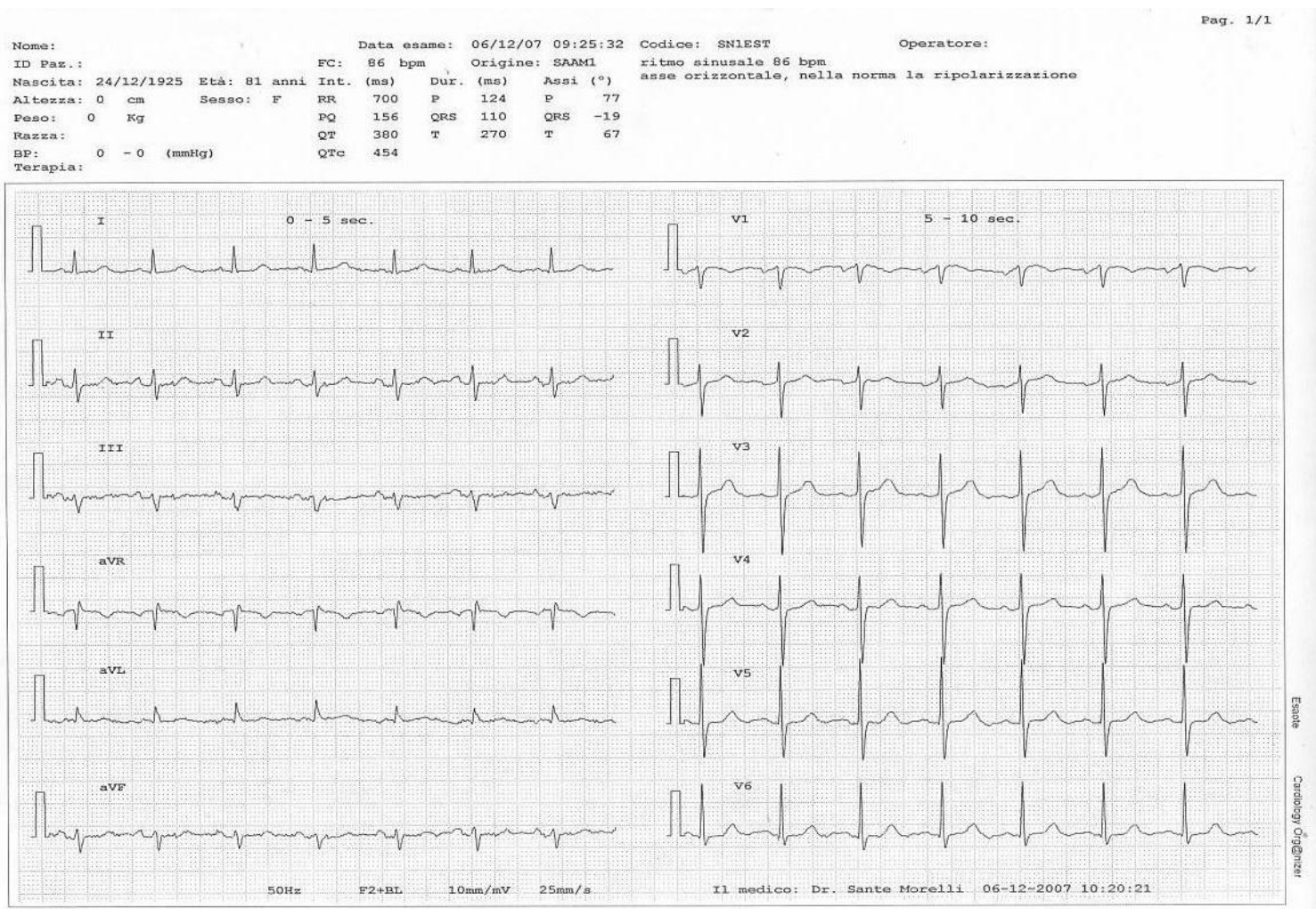

Picture 1. The electrocardiogram (2007) doesn’t show post-schemic alterations.
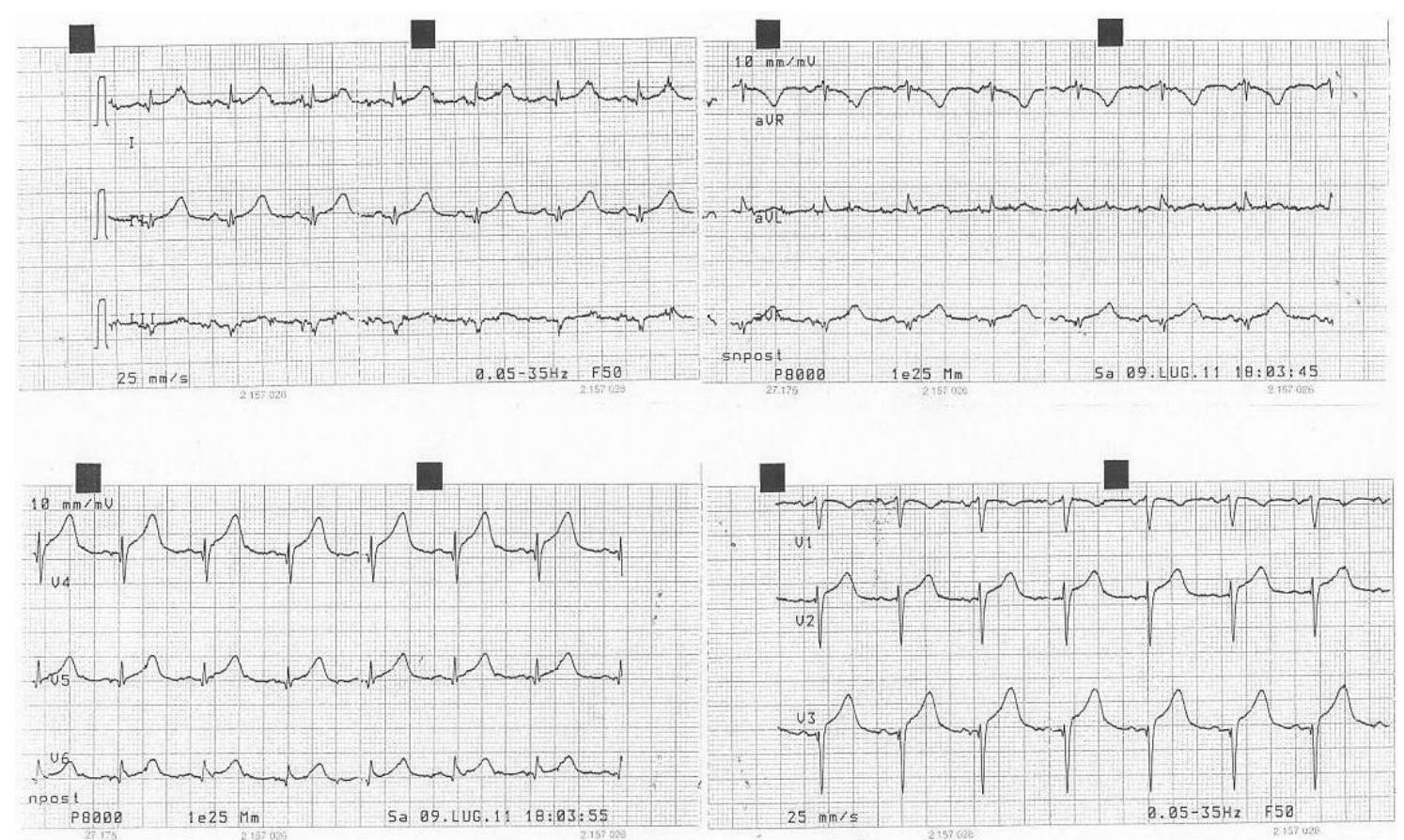

Picture 2. The electrocardiogram during thoracic pain, with ischemic alterations in anterior leads.

The coronarography showed haziness, but no critical stenosis; ventricolography revealed apical ballooning of the left ventricle with severe systolic dysfunction and apical thrombus. Table 2 shows blood test screening upon admission.

The patient received sodium enoxaparin $(4.000 \mathrm{U}$ b.i.d), intravenous infusion of furosemide (20 mg b.i.d) and started dopamine hydrochloride at $4 \gamma / \mathrm{Kg} / \mathrm{min}$ for 8 
Table 1. Intra arterial gas analysis.

\begin{tabular}{ccc}
\hline & & Normal Value \\
\hline $\mathbf{p H}$ & 7.427 & $7.37-7.45$ \\
$\mathbf{p C O}_{2}$ & $37.0 \mathrm{mmHg}$ & $35.0-46.0 \mathrm{mmHg}$ \\
$\mathbf{p O}_{2}$ & $101.2 \mathrm{mmHg}$ & $70.0-100.0 \mathrm{mmHg}$ \\
$\mathbf{H c t}$ & $34 \%$ & $36.0 \%-46.0 \%$ \\
$\mathbf{S O}$ & $98.4 \%$ & $>97 \%$ \\
$\mathbf{~ N a}$ & $130.3 \mathrm{mmol} / \mathrm{L}$ & $135.0-145.0 \mathrm{mmol} / \mathrm{L}$ \\
$\mathbf{K}$ & $3.82 \mathrm{mmol} / \mathrm{L}$ & $3.6-4.8 \mathrm{mmol} / \mathrm{L}$ \\
$\mathbf{C a}$ & $1.10 \mathrm{mmol} / \mathrm{L}$ & $1.15-1.35 \mathrm{mmol} / \mathrm{L}$ \\
\hline
\end{tabular}

Table 2. Blood test screening upon admission in cardiovascular ward.

\begin{tabular}{|c|c|c|}
\hline \multirow[b]{2}{*}{ White blood cells $\left(10^{9} / \mathrm{l}\right)$} & \multicolumn{2}{|c|}{ Normal Value } \\
\hline & 9.43 & $4.00-10.00$ \\
\hline Neu (\%) & 90.6 & \\
\hline Lyn (\%) & 3.3 & \\
\hline Mon (\%) & 5.9 & \\
\hline Eos (\%) & 0.0 & \\
\hline Bas (\%) & 0.2 & \\
\hline Red blood cells $\left(10^{12} / \mathrm{l}\right)$ & 3.4 & $4.00-5.20$ \\
\hline Haemoglobin (g/dl) & 9.7 & $12.0-15.5$ \\
\hline Hematocrit (\%) & 29.8 & $37.0-47.0$ \\
\hline Average red blood cell size (fl) & 87.6 & $80.0-95.0$ \\
\hline Haemoglobin amount per red blood cell (pg) & 28.5 & $27.0-32.0$ \\
\hline $\begin{array}{l}\text { Haemoglobin concentration per red } \\
\text { blood cell (g/dl) }\end{array}$ & 32.6 & $32.0-36.0$ \\
\hline Platelets $\left(10^{9} / \mathrm{L}\right)$ & 516 & $140-400$ \\
\hline Glycaemia (mg/dl) & 129 & $60-110$ \\
\hline Total Bilirubin (mg/dl) & 0.35 & $<1.00$ \\
\hline $\begin{array}{l}\text { Alanine aminotransferase/aspartate } \\
\text { aminotransferase (UI/l) }\end{array}$ & 10 & $<40$ \\
\hline Serum creatinine (mg/dl) & 0.76 & $0.70-1.20$ \\
\hline Serum sodium $(\mathrm{mEq} / \mathrm{l})$ & 133 & $135-146$ \\
\hline Serum potassium (mEq/l) & 4.4 & $3.5-5.3$ \\
\hline Total serum calcium (mg/dl) & 8.3 & $8.6-10.2$ \\
\hline Internationa normalized ratio & 1.77 & $0.8-1.20$ \\
\hline T-troponin & 903 & $<10$ \\
\hline
\end{tabular}

hours, raised to $8 \gamma / \mathrm{Kg} / \mathrm{min}$. A chest X-ray showed no pleuroparenchymal diseases. During the following two days the patient had stomach ache regressed by intravenous administration of lansoprazole; the electrocardiogram (Picture 3) showed a heart rate of $83 \mathrm{bpm}$, ST sopraelevation in $V_{3}$ and $V_{4}$, but she getting physically better and started to eat. The fourth day started the administration of bisoprolol $(2.5 \mathrm{mg} / \mathrm{die})$ and after 24 hours sodium warfarin to reach an INR target of 2.5.
Before the discharge of the patient to our ward was performed an hemodynamic and Doppler echocardiographic study of ventricular function which demonstrated normal size of the left ventricle, apical akinesis and ejection fraction decreased to $30 \%$, mild aortic insufficiency and apical thrombus. During hospitalization in our ward she was in sinus rhythm, apyretic and re-started physiotherapy; she was discharged 20 days later with appropriate home therapy. We performed a new echocardiographic study 90 day after the discharge, which confirmed the normal size of the left ventricle, the resolution of the apical thrombus and demonstrated the ejection fraction raised to $60 \%$ as shown in Picture 4. The patient is now at home in a fairly good condition.

\section{DISCUSSION}

The initial presentation of Takotsubo Syndrome is often indistinguishable from an acute coronary syndrome, so the patient commonly undergo urgent a coronary angiography as first test. Usually there are no significant stenoses [2] in the coronaries and the ventriculography reveals characteristic apical akinesis/dyskinesis with compensatory hyperkinesis of the basal segment of the left ventricle, but ventricular function improves 1 to 4 weeks afterwards in patients surviving the initial event. Patients commonly present with ST-segment elevation in the precordial leads [3], chest pain due to temporary stunning of the left ventricular apex and transient apical systolic left ventricular dysfunction. Cardiac markers are usually elevated, but the levels tend to be lower and normalize sooner than with acute coronary syndrome patients [4]. The precise etiology remains unknown [5], but proposed mechanisms include: multivessel coronary artery spasm or impaired cardiac microvascular function [6], and endogenous catecholamine induced myocardial stunning and microinfarction [7,8]. Acute complications occur in approximately $20 \%$ of Takotsubo cardiomyopathy cases and include cardiogenic shock, left-sided heart failure with or without pulmonary edema, tachy- and bradyarrhythmias, left ventricular thrombus formation or free wall rupture, and death $[4,9,10]$. The clinical importance of left ventricular thrombus, as in our case, lies in its potential to embolize and stroke continues to be a clinically important complication. Once thrombus has been identified, however, a long-term therapeutic anticoagulation, as we did, reduces the incidence of ischemic stroke. Anticoagulation and subsequent improvement in left ventricular function is associated with gradual resolution of apical thrombus. The long-term therapy of Takotsubo cardiomyopathy is that of cardiomyopathy with LV systolic dysfunction: beta-blockers, ACE-inhibitors, diuretics; aspirin and calcium channel blockers have also been recommended [10]. Prognosis is good for those who survive the acute episode and recovery time is generally 


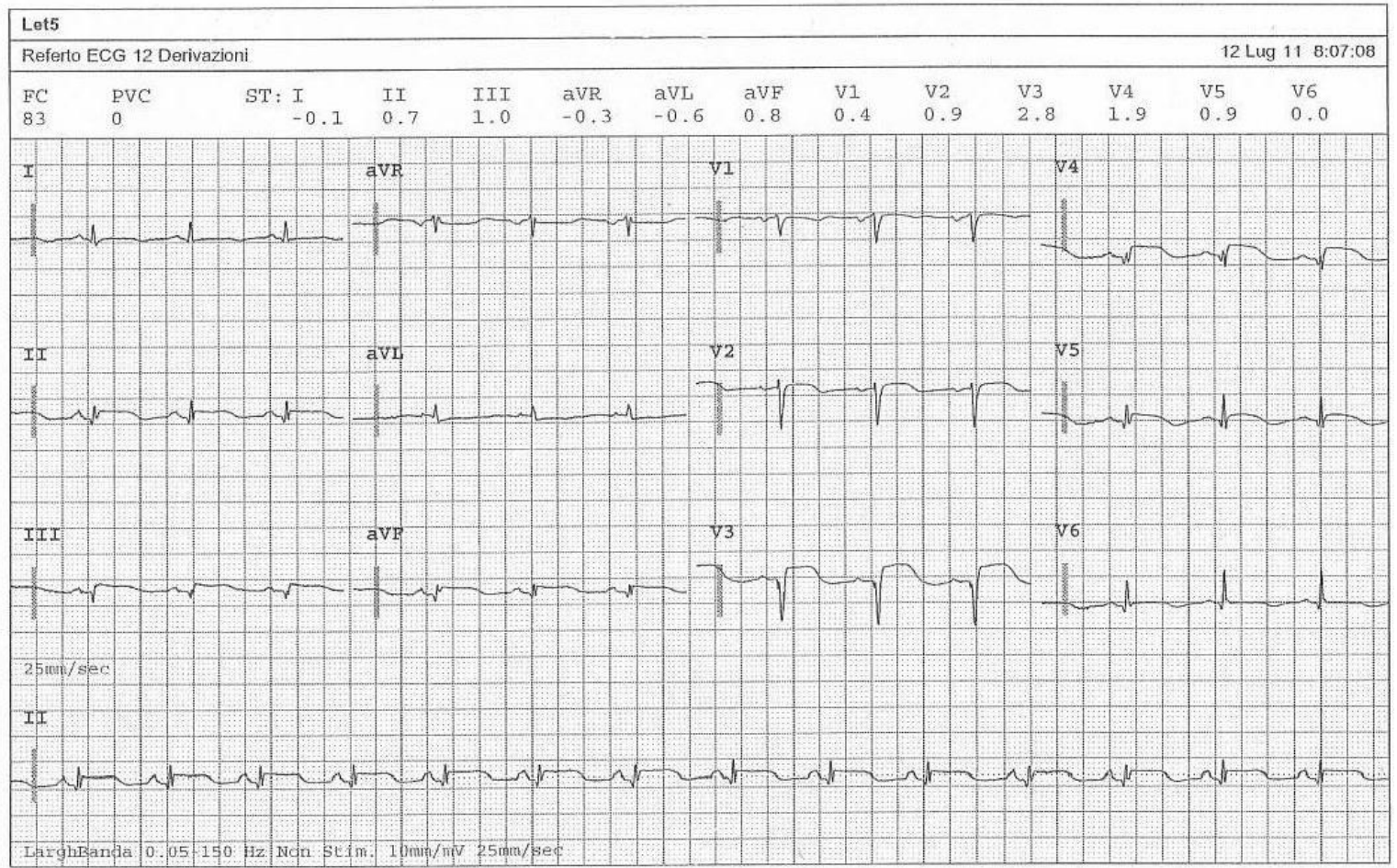

강 PHILIPS

Osp. Infermi Rimini Utic

Picture 3. The electrocardiogram in cardiovascular diseases department.

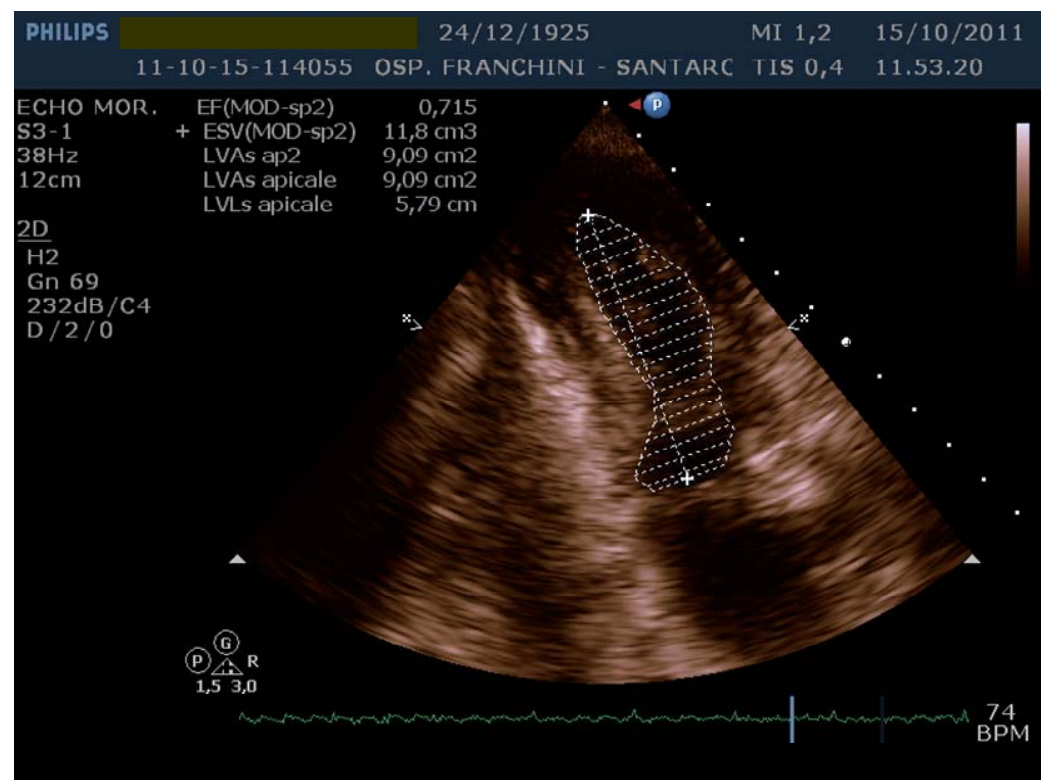

Picture 4. The ejection fraction raised to $46 \%, 90$ days after the discharge.

rapid.

Postmenopausal women account for 82 to 100 percent of cases. The syndrome may also occur in younger patients without cardiac risk factors. Some anxious or emotionally distraught patients with chest pain may be more complicated than simple anxiety disorder, and may de- velop dysrhythmias or shock: an episode of emotional or physiologic stress frequently precedes presentation with the syndrome. The emotional distress and chest pain are sometimes dismissed or managed with benzodiazepines and the association between onset of Takotsubo cardiomyopathy and emotional triggers is striking but has re- 
ceived little attention in the psychiatric literature [11] and in internal medicine. The close association between severe stress such as a surgical treatment and unfavorable physiologic outcomes is evidenced by our everyday use of such phrases as "broken heart," or "heart ache" however, transient cardiomyopathy associated with acute noncardiac pain is rare. Although Takotsubo cardiomyopathy has increasingly been reported in recent years, the prevalence is likely underestimated because of the low level of awareness and infrequent diagnosis. Increased awareness among physicians will improve recognition and clinical management and Takotsubo cardiomyopaty must be considered as a relevant differential diagnosis mimicking acute myocardial infarction.

\section{REFERENCES}

[1] Sato, H., et al. (1990) Takotsubo-type cardiomyopathy due to multivessel spasm. In: Kodama, K., Haze, K., Hon M, et al., Eds., Clinical Aspect of Myocardial Injury: From Ischemia to Heart Failure, Kagakuhyouronsha, Tpkyo, 56-64.

[2] Takagi, A. et al. (1999) Significance of angiographic haziness at the distal stent edge: Analysis by intravascular ultrasound and quantitative coronary angiography. American Journal of Cardiology, 33 , 307-316

[3] Kevin, A., Bybee, M.D. et al. (2004) Systematic review: Transient left ventricular apical ballooning: A syndrome that mimics ST-segment elevation myocardial infarction. Annals of Internal Medicine, 141, 11858-11865.

[4] Gianni, M., et al. (2006) Apical ballooning syndrome or takotsubo cardiomyopathy: A systematic review. European Heart Journal, 27, 1523-1529. doi:10.1093/eurheartj/ehl032

[5] Abe, Y., et al. (2003) Assessment of clinical features in transient left ventricular apical ballooning. Journal of the American College of Cardiology, 41, 737-742. doi:10.1016/S0735-1097(02)02925-X

[6] Bybee, K.A., et al. (2004) Clinical characteristics and thrombolysis in myocardial infarction frame counts in women with transient left ventricular apical ballooning syndrome. Journal of the American College of Cardiol- ogy, 94, 343-346. doi:10.1016/j.amjcard.2004.04.030

[7] Bybee, K.A., et al. (2008) Stress-related cardiomyopathy syndromes. Circulation, 118, 397-409. doi:10.1161/CIRCULATIONAHA.106.677625

[8] Wittstein, I.S., et al. (2005) Neurohumoral features of myocardial stunning due to sudden emotional stress. New England Journal of Medicine, 352, 539-548. doi:10.1056/NEJMoa043046

[9] Matsuoka, K., et al. (2003) Evaluation of the arrhythmogenecity of stress-induced "Takotsubo cardiomyopathy" from the time course of the 12-lead surface electrocardiogram. American Journal of Cardiology, 92, 230233. doi:10.1016/S0002-9149(03)00547-2

[10] Bybee, K.A., et al. (2004) Systematic review: Transient left ventricular apical ballooning: A syndrome that mimics ST-segment elevation myocardial infarction. Annals of Internal Medicine, 141, 858-865.

[11] Costin, G. et al. (2011) A psychosomatic perspective on takotsubo cardiomyopathy: A case report. Prim Care Companion CNS Disord, 13, 2. 